\title{
Antennal Lobe Processing Correlates to Moth Olfactory Behavior
}

\author{
Linda S. Kuebler, ${ }^{1}$ Marco Schubert, ${ }^{2}$ Zsolt Kárpáti, ${ }^{1}$ Bill S. Hansson, ${ }^{1 \star}$ and Shannon B. Olsson ${ }^{1 \star}$ \\ ${ }^{1}$ Department of Evolutionary Neuroethology, Max-Planck-Institute for Chemical Ecology, 07745 Jena, Germany, and ${ }^{2}$ Institute of Neurobiology, Free \\ University of Berlin, 14195 Berlin, Germany
}

\begin{abstract}
Animals typically perceive their olfactory environment as a complex blend of natural odor cues. In insects, the initial processing of odors occurs in the antennal lobe (AL). Afferent peripheral input from olfactory sensory neurons (OSNs) is modified via mostly inhibitory local interneurons (LNs) and transferred by projection neurons (PNs) to higher brain centers. Here we performed optophysiological studies in the AL of the moth, Manduca sexta, and recorded odor-evoked calcium changes in response to antennal stimulation with five monomolecular host volatiles and their artificial mixture. In a double staining approach, we simultaneously measured OSN network input in concert with PN output across the glomerular array. By comparing odor-evoked activity patterns and response intensities between the two processing levels, we show that host mixtures could generally be predicted from the linear summation of their components at the input of the AL, but output neurons established a unique, nonlinear spatial pattern separate from individual component identities. We then assessed whether particularly high levels of signal modulation correspond to behavioral relevance. One of our mixture components, phenyl acetaldehyde, evoked significant levels of nonlinear input-output modulation in observed spatiotemporal activation patterns that were unique from the other individual odorants tested. This compound also accelerated behavioral activity in subsequent wind tunnel tests, whereas another compound that did not exhibit high levels of modulation also did not affect behavior. These results suggest that the high degree of input-output modulation exhibited by the AL for specific odors can correlate to behavioral output.
\end{abstract}

\section{Introduction}

Natural odor cues are typically complex blends of diverse chemical compounds. In insects, odorant molecules are detected by olfactory sensory neurons (OSNs) and transduced into action potentials that input information about the odorant molecule to the brain. OSNs expressing the same olfactory receptor protein converge onto the same glomerulus in the antennal lobe (AL), the initial olfactory processing center and insect analog of the mammalian olfactory bulb (OB). Projection neurons (PNs) subsequently relay AL output to higher brain centers (for reviews see Homberg et al., 1989; Hildebrand and Shepherd, 1997; Hansson and Anton, 2000; de Bruyne and Baker, 2008).

Odors are represented as stable, spatial patterns of neuronal activity in the OB/AL, as shown by optophysiological studies in

Received Dec. 15, 2011; revised Feb. 7, 2012; accepted Feb. 28, 2012.

Author contributions: L.S.K., B.S.H., and S.B.O. designed research; L.S.K. and Z.K. performed research; L.S.K., M.S., Z.K., and S.B.O. analyzed data; L.S.K. wrote the paper.

This research was supported by European Union (EU) 6th Framework Programme FET Project iCHEM, the Max Planck Society, and by a Marie Curie IEF-255193 EU Grant to Z.K. We thank Daniel Veit for engineering the stimulus delivery system, writing the lab-view software, and solving technical problems. We are grateful to Silke Sachse for support and helpful advice on Calcium Imaging. We also thank Martin Strube-Bloss and Sonja Bisch-Knaden for statistical advice, Neloy Kumar Chakroborty for help on the odor trace analysis, and the iChem consortium for fruitful discussion.

*B.S.H. and S.B.O. share the last authorship equally.

The authors declare no competing financial interests.

Correspondence should be addressed to Shannon B. Olsson, Department of Evolutionary Neuroethology, MaxPlanck-Institute for Chemical Ecology, Hans-Knoell Strasse 8, 07745 Jena, Germany. E-mail: solsson@ice.mpg.de. DOI:10.1523/JNEUROSCI.6225-11.2012

Copyright $\odot 2012$ the authors $\quad 0270-6474 / 12 / 325772-11 \$ 15.00 / 0$ both vertebrates (Friedrich and Korsching, 1998; Rubin and Katz, 1999; Uchida et al., 2000; Meister and Bonhoeffer, 2001; Fried et al., 2002) and insects (Joerges and Neyer, 1997; Galizia et al., 1999b; Sachse and Galizia, 2002; Wang et al., 2003; Skiri et al., 2004; Carlsson et al., 2005; Silbering and Galizia, 2007). As a result of the separation of natural blend information into different OSN channels, subsequent integration of at least a subset of these channels is required to establish blend-specific features.

In recent years there has been considerable interest in studying artificial odor mixtures in invertebrates in regard to network processing (Deisig et al., 2006, 2010; Su et al., 2011; Joerges et al., 1997; Silbering and Galizia, 2007; Hillier and Vickers, 2011) and olfactory-guided behavior (Ditzen et al., 2003; Riffell et al., 2009b; Smith, 1998; Reinhard et al., 2010). Other studies have also addressed AL signal modulation in response to single odors (Bhandawat et al., 2007) and its relevance for motor output (Schlief and Wilson, 2007). However, few studies have combined these lines of inquiry to follow the modulation of complex odor mixtures in the AL from input to output, and its subsequent relationship to olfactory behavior.

In the current study, we applied optophysiological techniques to assess levels of spatiotemporal odor integration established by the AL of the hawk moth, Manduca sexta. Although processing in the pheromone-specific macroglomerular complex of the moth AL has been extensively studied (see recent review in Martin et al., 2011), it is unclear (Christensen and Hildebrand, 2002) how this specialized, labeled-line system (Galizia and Rössler, 2010) relates to the combinatorial processing found in the general, plantodor portion of the moth AL. By simultaneously applying two 
different calcium-sensitive dyes, we quantified the odor information fed into the network (OSN input) as well as the resultant output of network processing (PN output) triggered by a quintuple mixture of plant volatiles and its single components (Kuebler et al., 2011a). The double staining of input and output AL neurons in a single preparation allows direct assessment of input-output modulation without requiring topographical orientation to specific landmarks (i.e., glomeruli). The mixture compounds used were previously assessed in a single unit study of the Manduca AL (Kuebler et al., 2011b) and provide a chemically diverse excerpt of the odor bouquet of flowers and plants on which Manduca feeds and oviposits (Kessler and Baldwin, 2001; Allmann and Baldwin, 2010). In an attempt to correlate our physiological results with olfactory behavior, we subsequently tested components shown to undergo different levels of input-output spatiotemporal modulation in our optophysiological study with a known behaviorally attractive synthetic flower mixture (Riffell et al., 2009a). Our results indicate that complex host mixture processing in the moth olfactory system results in a unique mixture feature that cannot be predicted from peripheral input. Moreover, a comparison of optophysiological and behavioral results suggests that high levels of spatiotemporal signal modulation in the AL can correlate to behavioral activity, and offers evidence for a selective olfactory attention cue in moths. Our study bridges the gap between olfactory-guided behavior and neural correlates. It thus provides a novel, important insight into the establishment of behaviorally relevant odor features even at the first synapse of the insect CNS.

\section{Materials and Methods}

M. sexta (Lepidoptera, Sphingidae) larvae were reared in the laboratory on an artificial diet (Bell and Joachim, 1976). Female pupae were isolated individually in paper bags in an environmental chamber at $25^{\circ} \mathrm{C}$ with $70 \%$ relative humidity on a $16 \mathrm{~h} / 8 \mathrm{~h}$ light/dark photoperiod. Physiological and behavioral experiments were performed with naive adult females $3-5 \mathrm{~d}$ posteclosion.

\section{Optophysiology}

Odor stimulation. Each animal's antenna was exposed to a constant, charcoal-filtered air stream (compressed house air, 8 bar) regulated to a pressure of 2 bar and split into six independent channels, each controlled by a digital flowmeter with choke valve (SMC Pneumatik). Channels fed into a concentric arrangement of solenoid valves (3/2 way M5, Jenpneumatik) surrounding a central line, establishing the novel self-developed and custom-made stimulus delivery system (for detailed description of the multicomponent stimulus device, see Olsson et al., 2011). The resultant airflow passed through the central line and ended in a Peek nozzle (diameter $7 \mathrm{~mm}$ ) positioned $1 \mathrm{~cm}$ in front of the insect's antenna. Stimulus pulses were presented for $2 \mathrm{~s}$ and controlled with custom written software using Labview 8.5 (National Instruments). The quintuple odor mixture was obtained by switching on five valves simultaneously. A set of 11 bottles (two concentration stages per odor and mineral oil as control) were used to alter concentrations. To achieve the same relative odor concentrations during presentation, the vapor pressure of each component was considered and single component airflows were altered accordingly (Olsson et al., 2011).

The following odors were used: $(+)$-linalool $(+\mathrm{Lin}),(-)$-linalool (-Lin), phenyl acetaldehyde (PAA), trans-2-hexenyl acetate (E2HA) and cis-3-hexenyl acetate (Z3HA). All odors were acquired from Sigma at highest purity available and diluted in mineral oil at $1 \times 10^{-4}$, a concentration previously used for an electrophysiological study of the Manduca AL (see Kuebler et al., 2011b for rationale). Odors were selected because of their ecological and physiological relevance as shown in previous studies of M. sexta (King et al., 2000; Shields and Hildebrand, 2000; Fraser et al., 2003; Hansson et al., 2003; Reisenman et al., 2004; Kuebler et al., 2011b; Olsson et al., 2011). For optical recordings, stimuli were presented in a similar repetitive sequence across animals: We performed three experimental runs, first presenting each animal with a random stimulation sequence of each of the five single components and the control (mineral oil), followed by the complete quintuple mixture. Finally, animals were tested with the single components in random order at the appropriate "mixture" concentrations $\left(5 \times 10^{-4}\right)$ to compensate for concentration differences between the mixture and its components.

Dye loading and optophysiological recordings. We combined two approaches simultaneously in one animal, using two different calciumsensitive dyes: (1) bath application of membrane-permeable Calcium Green-2 AM (Galizia et al., 1998) monitoring neuronal activity dominated by afferent input to the AL (OSNs), and (2) retrograde selective staining of projection neurons (PNs) with Fura-2 dextran (Sachse and Galizia, 2002, 2003). For bath application, the membrane-permeable form of a fluorescent calcium indicator ( $30 \mu \mathrm{mol}$, Calcium Green-2 AM, Invitrogen) was dissolved in physiological saline (Heinbockel et al., 2004). The exposed brain and the preparation were incubated for $2 \mathrm{~h}$ at $4^{\circ} \mathrm{C}$ and rinsed afterward several times with physiological saline solution to remove excess dye.

Retrograde staining was performed by injection of dye into the PN antennocerebral tracts (ACTs) using a glass electrode coated with crystals of Fura-2 dextran (potassium salt, 10,000 MW, Invitrogen) dissolved in $4 \%$ bovine serum albumin solution. This dye application method has been shown to be specific and effective in honeybees (Sachse and Galizia, 2002). The glass electrode (diameter $15 \mu \mathrm{m}$ ) was manually inserted into the deutocerebrum close to the midline of both hemispheres, aiming for both the inner and outer antennocerebral tracts. Preliminary studies with tetramethylrhodamine-dextran (micro-Ruby, Invitrogen) were conducted to determine the location and projection of the different ACTs in Manduca. micro-Ruby was inserted into the AL using an identical injection procedure applied for Fura- 2 dextran. Dyes were allowed to travel for $2 \mathrm{~h}$ (micro-Ruby) and $7 \mathrm{~h}$ (Fura-2 dextran) at room temperature. Successful PN loading was determined by strong staining of the soma clusters (Fig. 1, asterisks).

Imaging was performed using a T.I.L.L. Photonics imaging system. Appropriate filter settings allowed simultaneous measurement of the two calcium-sensitive dyes. Monochromatic excitation light alternated between $340 \mathrm{~nm}, 380 \mathrm{~nm}$ (Fura-2), and $475 \mathrm{~nm}$ (Calcium Green). As filters, a dichroic beamsplitter (505DRLPXR) together with an $\alpha$ longpass emitter (emissionfilter, 515ALP) were used. Light emitted by stained cells peaked at wavelengths of $536 \mathrm{~nm}$ and $510 \mathrm{~nm}$ after excitation for Calcium Green-2 AM and Fura-2 dextran, respectively. The imaging set-up consisted of a CCD camera (SensiCam 672 KS 4683, PCO Imaging) mounted to an upright microscope (Olympus BX51WI) equipped with a waterimmersion objective (Olympus, $10 \times / 0.30$ ). Fourfold symmetrical binning resulted in $344 \times 260$ pixels images with one pixel corresponding to an area of $4 \times 4 \mu \mathrm{m}$. Single experimental trials were recorded with a sampling rate of $4 \mathrm{~Hz}$ for $10 \mathrm{~s}$, corresponding to $40 \times 250 \mathrm{~ms}$ frames for each of the two calcium-sensitive dyes. Light was shut off between frames. Stimulation began $2 \mathrm{~s}$ after recording onset for a period of $2 \mathrm{~s}$ (frames $8-16$ ). Several automated processing steps, e.g., background, bleaching, and movement corrections were applied using custom written software (IDL, ITT Visual Information Solutions) to enhance the signalto-noise ratio. Details of this data processing have been described in detail previously (Sachse and Galizia, 2002, 2003).

Raw data processing and attribution of activity patterns. In this study, the olfactory response to an odor mixture versus its single components was directly compared at two different processing levels (OSNs and PNs) within a single animal. Olfactory responses leading to increased neural activity were monitored as spatially restricted activity regions of increased fluorescence (F) in the AL. Fluorescence input signals (Calcium Green-2 AM, $475 \mathrm{~nm}$ measurements) were calculated as $\left(\mathrm{F}-\mathrm{F}_{0}\right) / \mathrm{F}_{0}$ $(\Delta \mathrm{F} / \mathrm{F})$ with background $\mathrm{F}_{0}$ as the average of florescence in frame 3-7. Output signals (Fura-2 measurements) were first calculated as the ratio of the emitted light $(340 \mathrm{~nm} / 380 \mathrm{~nm}$, multiplied by 1000) and then compared as $\Delta \mathrm{F} / \mathrm{F}$. Fura-2 ratios are approximately proportional to changes of the intracellular calcium concentration (Sachse and Galizia, 2002, 2003) 
A

Mixture
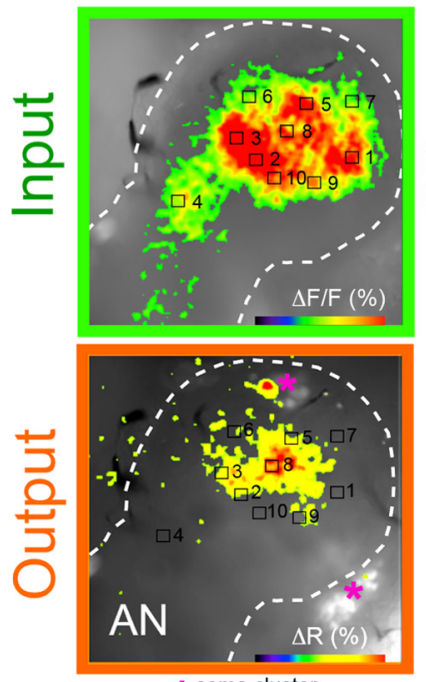

C
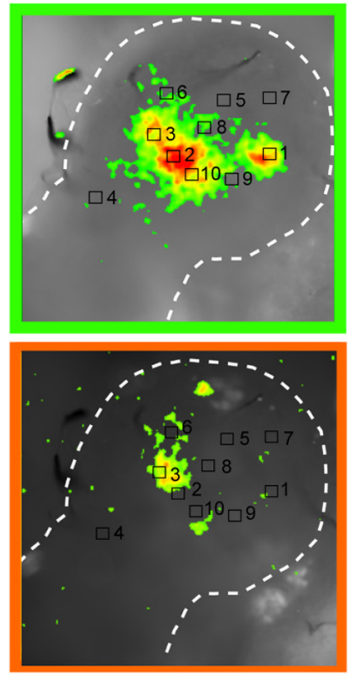

$(+)$ Lin

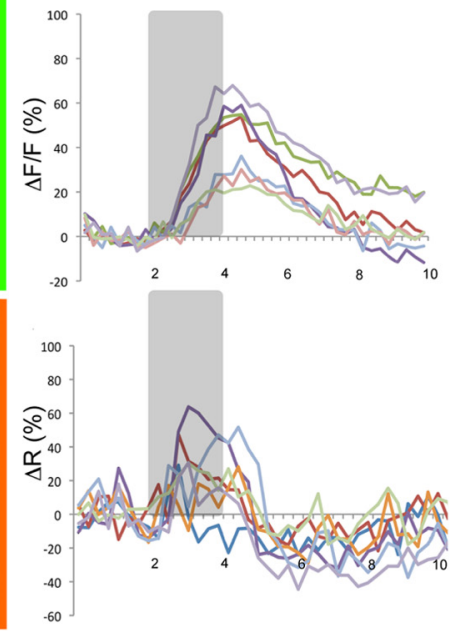

E

Z3HA
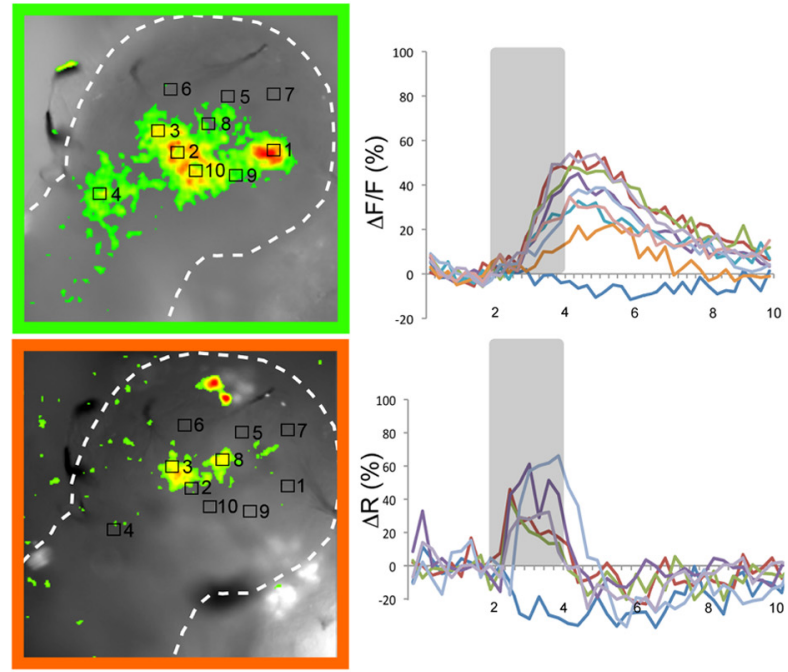

B

PAA
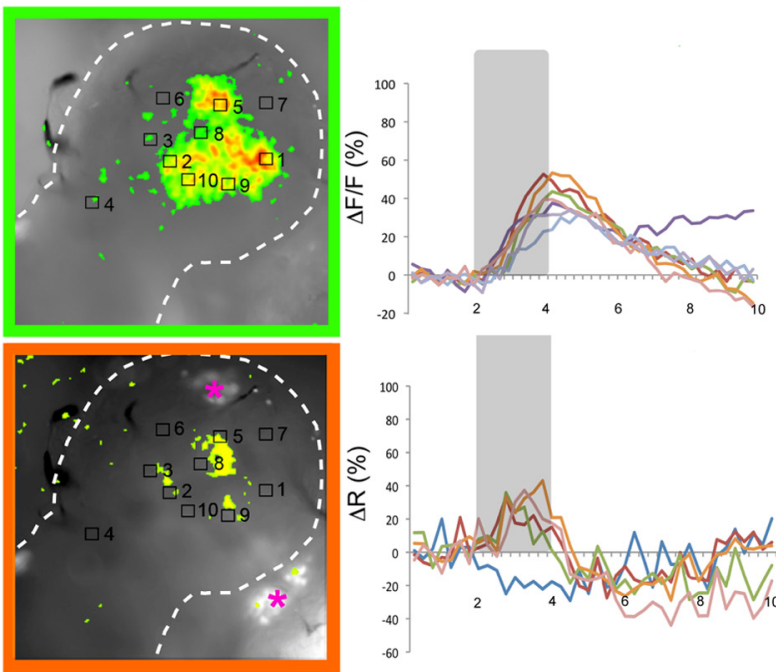

D

$(-)$ Lin
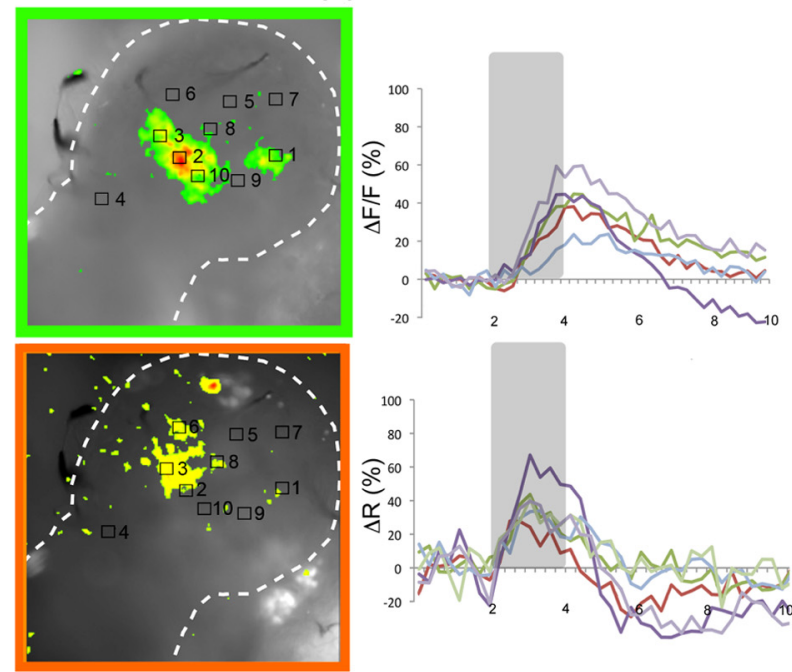

F

E2HA
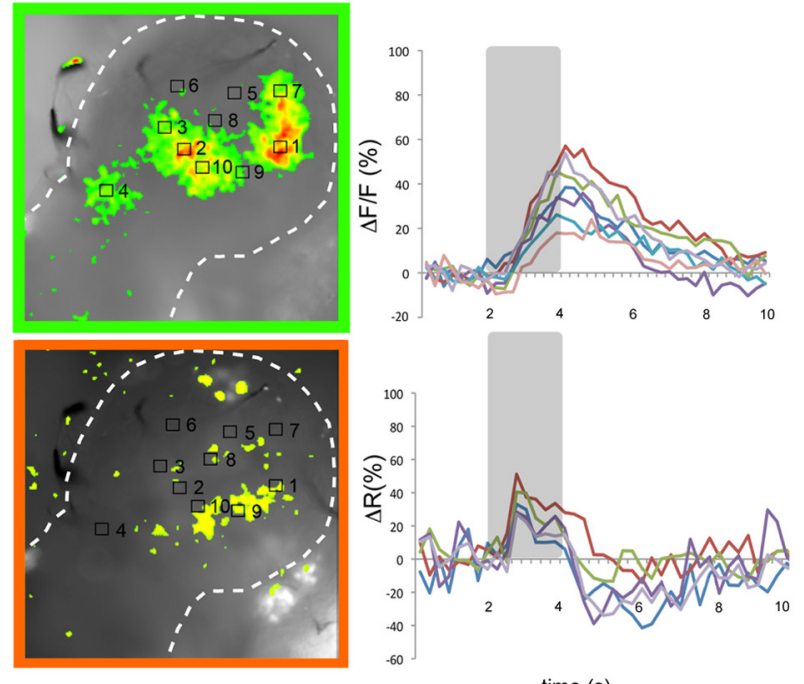

Figure 1. 

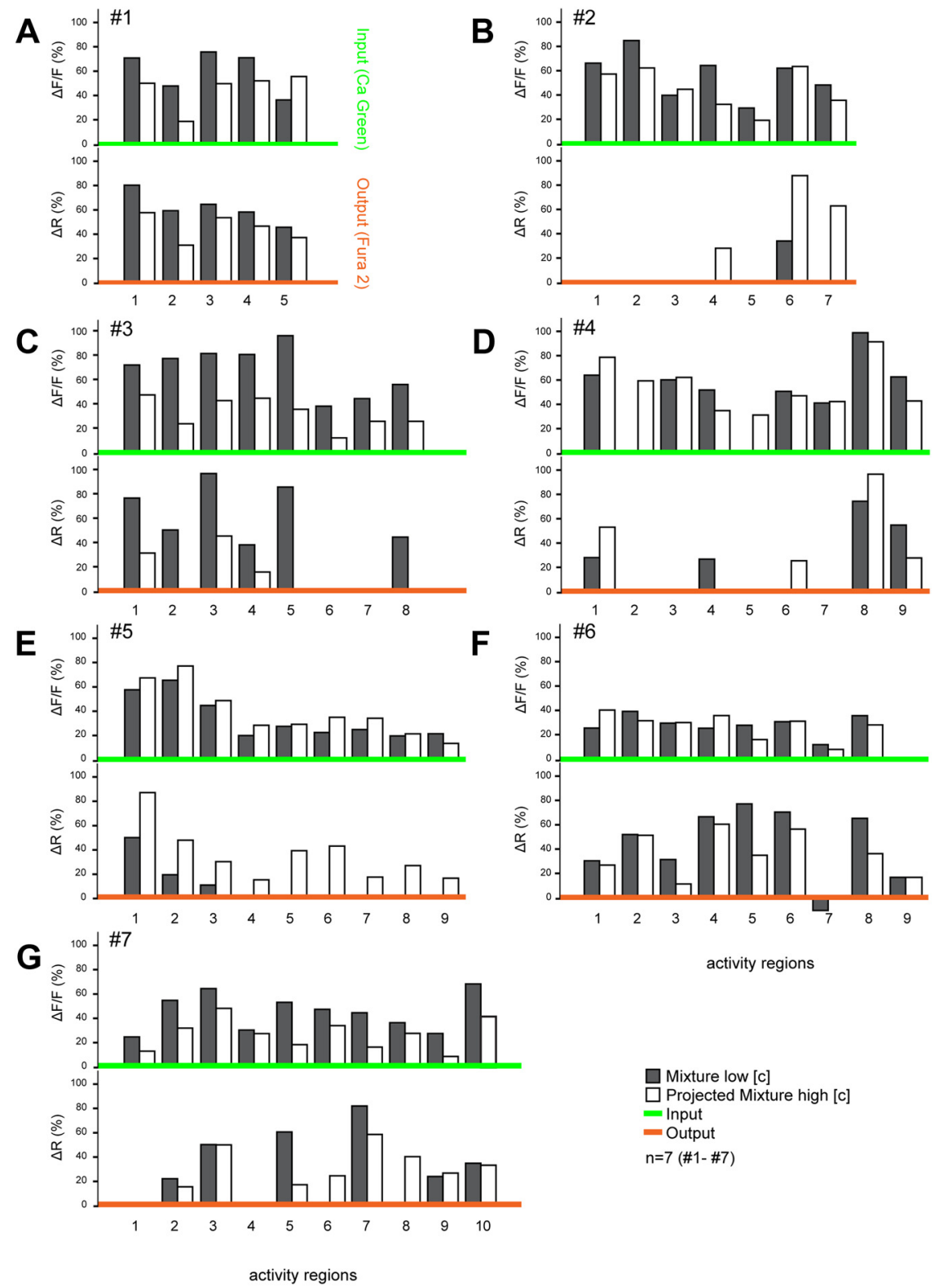

activity regions

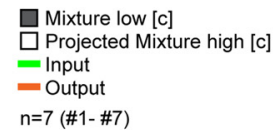

Figure 2. Comparison of mixture representations in the AL at both input and output processing levels shows strong interactions in PNs suggesting network modulation. Black bars depict the normalized response activity to the mixture for each activity region (number differs among animals $\boldsymbol{A}-\mathbf{G}$ from 5 to 10 ). To assess the predictability of response patterns, a projected mixture for each animal was created (white bars) using the maximum fluorescence change in all activity regions across every single component response tested at the mixture concentration (input and output respectively). Comparison of the measured mixture response (black bars) with the projected mixture (white bars) within animals showed that the mixture representation could generally be predicted from the response patterns of the single components at the input level (green $x$-axis, upper trace). PN mixture responses (output level, orange $x$-axis, lower trace) showed substantial loss and gain of activity regions compared with single component patterns. Thus, output patterns of single components did not typically reassemble the mixture response.
Input and output signals were attributed to regions of high activity. To compare both levels within individual animals, we had to compensate for different background fluorescence and maximal activities by setting the strongest response region of each animal to $100 \%$ and scaling each response accordingly. In each animal the odor responses were normalized to the maximal response for each dye separately [ $\max$ $\Delta \mathrm{F} / \mathrm{F}$ for Calcium Green, $\max \Delta \mathrm{R}(340 \mathrm{~nm} / 380$ $\mathrm{nm}$ ) for Fura-2]. Maximal responses measured in each activity region had to exceed $2 \times \mathrm{SD}$ above noise level for excitatory and $1 \times$ SD for inhibitory responses. Noise was determined as the SD of the spontaneous activity between frames 3-7 (before stimulus onset) and averaged across all measurements within one animal. The applied differences in response criteria were necessary because inhibitory signals in general displayed weaker intensities than excitatory signals.

Although a given focal plane of the Manduca AL would reveal 10-15 glomeruli (cf. GrosseWilde et al., 2011), single glomeruli could not be visually identified under fluorescence light. Thus regions of activity were set and analyzed separately for each individual. Coordinates were established to mark the center of activity regions and to superimpose square areas of interest in which the mean activity was calculated over $11 \times 11$ pixels covering an area of $44 \times 44$ $\mu \mathrm{m}$. The areas of interest corresponded to $50 \%$ of an averaged sized AL glomerulus in Manduca (Grosse-Wilde et al., 2011). We assigned individual coordinates to $5-10$ activity regions per animal. False-color pictures display the change in fluorescence between two single frames, frame 4 (background) and 15 (response) for both processing levels. A threshold was set to show only active regions and remove noise.

Input/output comparison and measurement of odor similarity in the moth AL. Odor-evoked calcium changes in OSNs and PNs of 8 animals were analyzed. For each trace, regions of statistically significant activity were defined as coordinates in an $n$-dimensional space ( $n$ defined by the number of coordinates, ranging from 5 to 10, Fig. 2, Galizia et al., 1999a; Deisig et al., 2003; Sandoz et al., 2003; Deisig et al., 2006). Direct pattern comparison between individuals would require landmark (i.e., glomeruli) identification, which was not accessible in the Manduca preparation. To quantify the similarity of resultant odor-evoked activity patterns between individuals without the need to compare any landmarks, a vector was calculated for each trace within an individual using

Figure 1. Representative example of simultaneous imaging within a single Manduca female AL. Olfactory responses to an odor mixture versus its single components leading to increased neural activity were monitored as spatially restricted activity regions of fluorescence change $(\Delta \mathrm{F}$ ) in the AL at two different processing levels (input, compound signal, green frame and output, PNs, orange frame). $\boldsymbol{A}-\boldsymbol{F}$, Left, False color coded AL patterns evoked by the mixture and its single components are overlaid on the intrinsic fluorescent background (note that individual AL glomeruli are not visible in the preparation). Clear spatial differences in odor-evoked activity patterns between input and output indicate AL network modulation. The AL border is marked with a dotted line with the antennal nerve (AN) entering from the lower left corner. Magenta asterisks in A and B indicate stained PN soma clusters. Note that the glomerular structure is not visible. $\boldsymbol{A}-\boldsymbol{F}$, Right, Time course of calcium signals in individual activity regions. Each recording trial lasted $10 \mathrm{~s}$ (40 frames) with odor stimulation ( $2 \mathrm{~s}$ ) indicated by gray bars. With both staining methods, odor application lead to a strong change in calcium levels. Odor responses were normalized to the maximal response for each dye separately. PN responses showed a characteristic faster rising and decay time than the prolonged compound signals. Note that at the output level, odors evoked both excitation and inhibition in PNs (e.g., $\boldsymbol{B}, \boldsymbol{E}$, inhibitory signals in activity region 7). 
all responding coordinates during a given time window (frame 12-18). The vector was established from the dot product of activity regions between two traces. For example, the dot product between traces $\mathrm{a}$ and $\mathrm{b}$ for an animal with 5 activity regions would be calculated as:

$$
\begin{aligned}
A \cdot B=\left(a_{1}+b_{1}\right) & +\left(a_{2}+b_{2}\right)+\left(a_{3}+b_{3}\right) \\
& +\left(a_{4}+b_{4}\right)+\left(a_{5}+b_{5}\right)
\end{aligned}
$$

where $1-5$ are the activity regions of traces a and $\mathrm{b}$ (e.g., regions in Fig. 2), and $\mathrm{A}$ and $\mathrm{B}$ are the resultant vectors. From the dot product, the angle between the vectors of the two traces could be calculated from [acos (dotproduct)] and converted to a unit vector for graphing and analysis. Thus, compared traces with similar spatial responses would generate angles close to $0^{\circ}$, while traces with exclusive patterns would establish an angle of $90^{\circ}$ (Fig. 3A). In this manner, patterns across individuals could be compared as angles without requiring a direct comparison of topographical coordinates across all tested animals.

To compare the evolution of spatial patterns over time, activity regions for specific stimuli were compared across individuals by converting the activity pattern into correlation matrixes. In each matrix, cells represent the correlation to its adjacent horizontal and vertical neighbor. The matrices are symmetrical and span in both dimensions over time (frames). Response patterns were correlated from one time frame to the next over the entire $10 \mathrm{~s}$ experiment, including the time before, during and after odor stimulation. The correlation between odor pair response patterns over time (Fig. $3 B$ ) was determined by using a sliding window Pearson's correlation ranging from -1 (anticorrelated) to 1 (correlated, cf. Szyszka et al., 2011; Galili et al., 2011).

\section{Behavioral experiments}

Wind tunnel assays. The behavioral activity of $\mathrm{PAA}$ and $\mathrm{Z} 3 \mathrm{HA}$ was tested in a wind tunnel assay. The laboratory wind tunnel (Plexiglas, length $\times$ width $\times$ height $=3 \mathrm{~m} \times 1 \mathrm{~m} \times 1 \mathrm{~m}$ ) was set to an airflow of $0.4 \mathrm{~m} \mathrm{~s}^{-1}, 0,5$ Lux light source from top, $23^{\circ} \mathrm{C}$, and $70 \% \mathrm{RH}$. Laminar airflow was created by a $1 \times 1 \mathrm{~m}$ wire mesh mounted between an activated charcoal filter and the source. Green dots (diameter $5 \mathrm{~cm}$ ) were placed randomly in a nonoverlapping pattern on the floor of the wind tunnel to provide optomotor cues. Naive females (hungry, virgin, no olfactory experience) were tested individually to a single stimulus. Two hours prior behavioral testing, individual females were transferred from their rearing bags to a netted releasing tube $(15 \times 22 \mathrm{~cm})$ and moved into the wind tunnel room. This procedure allowed the animals to acclimate to ambient conditions in the flight tunnel. For testing, the tube was placed on a release platform at the downwind end of the tunnel, $50 \mathrm{~cm}$ above the floor and $260 \mathrm{~cm}$ downwind from the source. All experiments were conducted within the first $3 \mathrm{~h}$ of scotophase. Females were tested individually for a maximum of $5 \mathrm{~min}$. The following behav-
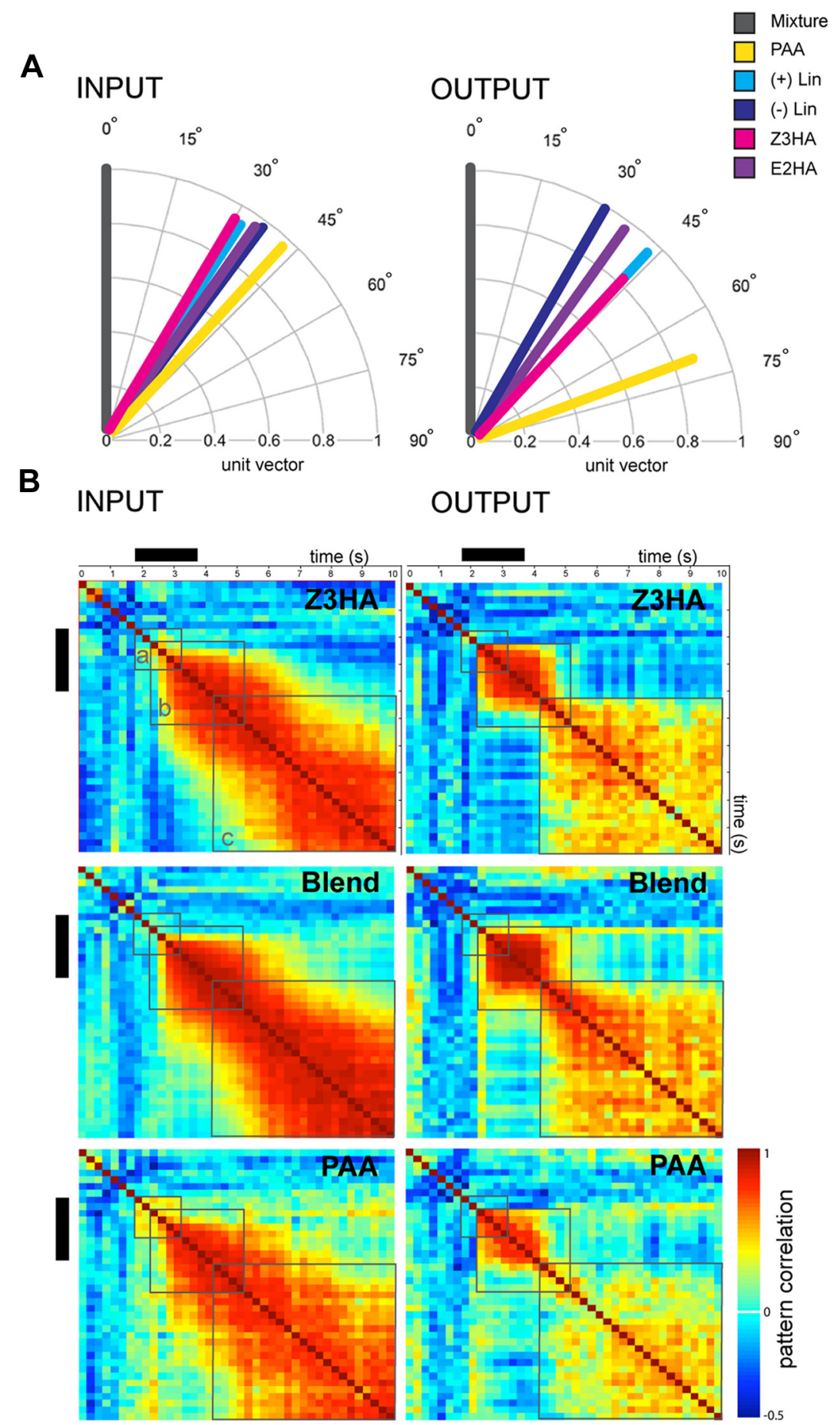

a early odor response, $\quad$ b odor \& early trace response, $C$ trace response

Figure 3. Odor pattern similarity in the Manduca $\mathrm{AL}$ at two separate processing levels. $A, 0$ dor representation changes from input to output in the AL network ( $n=8$ for input, $n=7$ for output). A resultant vector for each response pattern was calculated, and the dot product between vectors was used to determine the angle between mixture and single component responses tested at the mixture concentration [acos (dotproduct)]. Comparison of the mean angle of each of the single components compared with the mixture shows a spread at the PN level indicating network interactions at the output, most obvious for PAA. $B, 0$ dor response traces differ between OSNs (INPUT, $n=8$ ) and PNs (OUTPUT, $n=7$ ), and are odor specific. Correlation matrices depict the similarity of response patterns between measurements over time for selective odor stimuli at the mixture concentration. Response patterns are calculated as vectors with signal values of 66 activity regions for input and output responses, respectively. Each pixel represents the pattern correlation between two adjacent time points. PAA exhibits the most obvious spread and minimum pattern similarity over time not only in the time after odor onset (black bar) but across the entire $10 \mathrm{~s}$ recording. Similarity between response patterns was tested using a sliding window Pearson's correlation. Gray boxes indicate areas assessed for the ( $\boldsymbol{a}$ ) early odor response, $(\boldsymbol{b})$ odor and early trace response, and (c) trace response. Black bars indicate stimulus onset (2 s odor pulse). 
ioral response characteristics were observed: (1) time to wing fanning, i.e., preparation for flight (Fraser et al., 2003), (2) time to flight, (3) time to source contact. Upwind orientation was characterized by an anemotactic flight path. Both time and total number of animals performing the behavioral characteristics named above were counted.

Odor sources. Five different sources were tested: (1) synthetic flower mixture [Flower Mix: benzyl alcohol 90\%, \pm linalool 7\%, benzaldehyde 3\%; (Riffell et al., 2009a) ]; (2) Flower Mix + PAA: (benzyl alcohol 89.7\%, \pm linalool $6.7 \%$, benzaldehyde $2.7 \%$, phenyl acetaldehyde $1 \%$ ); (3) phenyl acetaldehyde 1\%; (4) Flower Mix + Z3HA: (benzyl alcohol 89.7\%, \pm linalool $6.7 \%$, benzaldehyde $2.7 \%$, cis-3-hexenyl acetate $1 \%$ ); (5) mineral oil solvent. A $1 \%$ concentration was chosen for PAA and Z3HA so that it did not exceed the concentration of any of the initial mixture components. $1 \times 2 \mathrm{~cm}$ filter paper sources were prepared by placing 10 $\mu \mathrm{l}$ of the different synthetic mixtures or solvent (e.g., $142 \mu \mathrm{g}$ of total Flower Mix). The loaded filter paper was placed in the wind tunnel 10 min before experiment onset. A total of 140 moths were tested. Note that the Flower Mix (Riffell et al., 2009a) was used because the chemically diverse quintuple mixture analyzed in the optophysiological studies (composed of PAA, Z3HA, E2HA, + Lin and - Lin) did not elicit significant upwind flight in Manduca females itself.

\section{Statistical analysis and figures}

Statistical analyses were performed using PASW 18.0 (SPSS, IBM) and R (R Development Core Team, 2011) statistical packages. Correlation matrices were performed using customized MATLAB (Mathworks) codes and tested for significance using the Mantel test (1000 random permutations, Mantel, 1967). Circular statistical analyses (parametric WatsonWilliams $F$ test to compare mean angles from two populations) were performed using Origin (OriginLab). Scientific artwork was compiled for publication with Adobe Photoshop and Illustrator CS4 (Adobe Systems).

\section{Results}

To assess the neural mechanisms of complex olfactory processing in the antennal lobe (AL) of Manduca sexta, we analyzed AL responses to 5 monomolecular odorants and their quintuple mixture. Odor-evoked calcium changes were recorded simultaneously for input (compound signal, dominated by OSN activity; Sachse and Galizia, 2002, 2003) and projection neuron (PN) output in a single preparation. This combined physiological approach enabled us to directly measure how the cellular network of the AL shaped the spatial representation of an odor mixture compared with its single components. Subsequent wind tunnel experiments testing odor-evoked flight activity revealed behavioral correlates to observed spatial activity patterns.

\section{Spatiotemporal response patterns}

Odor-evoked calcium changes to monomolecular odorants and their quintuple mixture lead to specific spatial activity patterns in the AL. In our study, 7 moths (of 140 attempted stained animals) showed clear, reliable calcium responses at both processing levels and allowed us to record complete stimulus protocols. An eighth moth provided only input responses and was used strictly for input analyses shown in Figure 3.

Two-second odor pulses led to consistent excitatory responses (intracellular calcium increase) resulting in odor-specific activity patterns at the input level (OSNs, compound signal, Fig. 1, green). Most recorded $\mathrm{PN}$ responses were excitatory, however in $\sim 10 \%$ of the output responses, the $\Delta \mathrm{R}$ decreased significantly reflecting an inhibitory response to the odor (intracellular calcium decrease in 6 of 7 animals; Fig. $1 B, E$, Fig. $2 F$ ). Successful dye loading in PNs could be easily determined by staining of the soma clusters (Homberg et al., 1988; Fig. 1A,B asterisks) and single somata showed strong excitation and/or inhibition to odor stimuli (Fig. 1, excited somata). The mixture and its single com- ponents induced signals in different combinations and numbers of activity regions, ranging from 5 to 10 between individuals. Each activity region covered an area of $44 \times 44 \mu \mathrm{m}$, corresponding approximately to $50 \%$ of an average sized AL glomerulus in Manduca (Grosse-Wilde et al., 2011). In general, the very strong mixture response resulted in a much broader pattern when compared with the single components at both processing levels (e.g., 10 activated regions in Fig. 1). Overall, activity regions at the output level showed complex odorant-dependent response patterns. The spatially restricted activity patterns observed at the output level here correspond well to the sparse response of PNs to these same volatiles in a previous electrophysiological study $(1 / 20$ responsive neurons; Kuebler et al., 2011). Figure 1 shows that the various stimuli elicited clear spatial differences in odor-evoked activity patterns between input and output indicating AL network modulation.

Odor-evoked PN (output) responses showed faster temporal dynamics when compared with the simultaneously measured compound signals (input) in all animals (output: $1.6 \pm 1.6 \mathrm{~s}$ SEM to reach max after stimulus "mixture" onset vs input: $2.7 \pm 0.7 \mathrm{~s}$ SEM, $n=7)$. The differences in temporal dynamics between input and output timing are possibly due to differences in dye dynamics or to differences in the calcium influx and buffering mechanisms of PNs and OSNs (Sachse and Galizia, 2003). In general, Calcium Green input signals showed a phasic-tonic shape, with a slow return to baseline after stimulus offset and in some cases a prolonged tonic component with no return to baseline within the $10 \mathrm{~s}$ recording $(>7 \mathrm{~s}$, Fig. $1 A, C, E, F)$. In contrast, Fura-2 output recordings were clearly phasic, returning to baseline very quickly ( $\sim 2.5 \mathrm{~s}$ after stimulus onset, Fig. 1$)$. Thus PN responses showed a characteristic faster rising and decay time when compared with the prolonged compound signals. Because of these observed differences in temporal kinetics between signals, we do not compare temporal modulation between AL input and output further. However, these differences do not prohibit the assessment of spatial patterning, which we discuss below.

\section{Input/output comparison reveals AL network modulation}

Emergent mixture patterns between the two processing levels

To assess the predictability of the mixture response patterns, a hypothetical mixture representation was created for each animal from the linear summation of patterns for all individual components (Fig. 2, white bars). The maximum fluorescence change in all activity regions across every single component response was set as the predicted mixture response for each individual animal (input and output, respectively). Although a glomerular map of Manduca sexta has recently been published (Grosse-Wilde et al., 2011), Figure 1 shows that Manduca sexta glomeruli are not intrinsically apparent during imaging. It is also difficult to maintain the same focal plane when attempting postimaging staining of glomeruli. As our goal was to assess levels of input/output AL modulation within individuals rather than identify specific glomerular activity patterns across individuals, we chose not to assign the activity regions to specific glomeruli, but rather emphasize within-individual differences (Fig. 2).

A comparison of the recorded mixture patterns (black bars) with the projected mixture (white bars) at the input level (top trace, green $x$-axis) showed similar patterns in 6/7 animals. This indicates that the mixture representation of the compound signal could generally be predicted from the response patterns to the individual components, and suggests that no strong network modulation at the OSN level took place. However, in one single case the mixture response showed less activity regions than pre- 
dicted by the single component patterns (Fig. $2 D$, activity regions 2 and 5) suggesting possible, but rare, network feedback processes already at the input level.

In contrast, a comparison of measured and predicted mixture patterns at the output level indicates strong network interactions, even when controlling for concentration between single components and the mixture (see Materials and Methods, Optophysiology, Odor stimulation). We differentiated between two main network interaction types, synergism and suppression (Duchamp-Viret et al., 2003; Silbering and Galizia, 2007; Kuebler et al., 2011b). Our criteria were highly restrictive: to be considered as a suppressive interaction, at least one of the activity regions in the mixture pattern (black bars) had to completely disappear compared with the projected mixture (white bars). In turn, to match the criteria for a synergistic response a new activity area (black bar) had to be recruited. Following these simple rules, on the output level 4/7 animals showed strong suppressive effects in the PN response (Fig. $2 B, D, E, G$ ). Synergistic effects were less common but were found in 3 animals (Fig. $2 C, D, F$ ). As a result, the mixture response patterns of output neurons could not be predicted from the single component responses in 6/7 animals. Areas that were highly activated by single components disappeared completely in the mixture pattern and vice versa (Fig. 2, except A). Comparison between the two processing levels revealed predominantly suppressive effects from input to output (6/7 animals). Synergism was witnessed in only one animal. In this case, in addition to a clear suppressive effect (Fig. $2 F$, inhibition in region 7) a new activity region was recruited that was not active at the input level (synergism, area 9).

\section{Odor response patterns between the two processing levels}

To more thoroughly assess how odor representations changed from input to output, we converted the spatial neural representation of each odor to a vector (Fig. $3 A$, see also Materials and Methods, Optophysiology, Input/output comparison and measurement of odor similarity in the moth $\mathrm{AL}$ ). This simple approach offers a comparison of patterns across tested individuals without the need to define common coordinates (i.e., glomeruli) between individuals. Figure $3 A$ compares the mixture (gray) mean angle versus its single components across all animals at both processing levels, input (OSNs) and output (PNs), respectively. The clear spread in vector angles at the output level, most obvious for phenyl acetaldehyde (PAA, yellow vector), indicates substantial network interactions when compared with the input. Whereas in most cases single components diverged from the mixture and separated more in the output (also compare Fig. 1), only PAA (yellow vector) was found to change in pattern between input and output when compared with the mixture (mixture/PAA input vs output, $p=0.08$ vs $p>0.3$ for all other compounds, $F$ test, Fig. $3 A$ ). Further comparisons between single component patterns (testing all possible odor pairs) show that only PAA elicited significantly different response patterns from the other 4 components tested. Input patterns evoked by PAA were significantly different with respect to cis-3-hexenyl acetate (PAA/Z3HA $p=0.03$; Watson-Williams $F$ test). At the output level, PAA response patterns were significantly different from all single components tested $(\mathrm{PAA} /+\operatorname{Lin} p=0.01, \mathrm{PAA} /-\operatorname{Lin} p=$ $0.008, \mathrm{PAA} / \mathrm{E} 2 \mathrm{HA} p=0.01, \mathrm{PAA} / \mathrm{Z} 3 \mathrm{HA} p=0.025$; WatsonWilliams $F$ test). Together, PAA clearly shows decreased pattern similarity from input to output indicating strong network interactions - not only when compared with the mixture but also to each single component itself.
Spatiotemporal activity patterns show strong odor-specific

differences

We next analyzed the spatiotemporal dynamics of the OSN (input) and PN (output) odor and post-odor response for the entire $10 \mathrm{~s}$ recording period. In contrast to Figures 1 and 2, which depict odor-evoked activation patterns in a time window right after stimulus onset showing the maximal responses, Figure 3 demonstrates an analysis in which we investigated the evolution of these activation patterns over the whole experimental timeline (including the activity before, during and after stimulus presentation).

By visualizing the time trace of the activity pattern, we were able to compare intensity and stability of the patterns. Thus, high levels of correlation indicate that the patterns progressed consistently over time. Figure $3 B$ shows a correlation matrix providing information about the time-resolved correlation within odor response patterns for both input (left) and output responses (right) across all tested animals $(n=8)$. Comparison of full time traces for PAA (lower trace, the compound that revealed the least pattern similarity to the mixture; Fig. $3 A$, yellow vector) and $\mathrm{Z} 3 \mathrm{HA}$ (upper trace, the only compound with significantly different response patterns from PAA at both processing levels; Fig. $3 \mathrm{~A}$, magenta vector) were compared with the mixture pattern itself (middle trace). Colored squares indicate the level of correlation in odor patterns between specific time points ( $250 \mathrm{~ms}$ bins), from maximum (red), to minimum (light blue), to anticorrelation (blue). To assess the pattern correlation between input and output responses all possible matrices where compared by testing different time intervals using a random permutation test (Mantel, 1967, Fig. $3 B a$, early odor response; $B b$, odor and early trace response; $B c$, trace response). The mixture response showed a high correlation of patterns and thus, response patterns were significantly correlated between input to output over time (Mantel test, mixture $r=0.8, p=0.001)$. The input and output response patterns for Z3HA were also significantly correlated (Z3HA $r=0.42, p=0.05$ ). Indeed, only PAA response patterns were not significantly correlated during the odor and early trace response from input to output (PAA $r=0.26, p=0.10$, for sector $\mathrm{b}$ odor and early trace response, testing input vs output). Thus, the apparent decorrelation of the PAA response pattern between input and output was not only present during odor stimulation (Fig. 3A) but was reflected across the whole recording.

\section{High levels of input-output modulation correlate to behavior}

We then asked whether the observed differences in spatiotemporal processing between input and output for different odorants have any correlation to olfactory behavior in Manduca sexta. To test behavioral correlates to AL network processing, we conducted wind tunnel experiments to examine upwind flight and source contact behavior of moths to PAA, the compound exhibiting the highest levels of spatiotemporal modulation between input and output, and Z3HA, a compound exhibiting significantly lower levels of input-output modulation compared with PAA.

Neither PAA alone (Fig. 4 yellow bar), nor the chemically diverse mixture of host floral and plant volatiles used in our optophysiological studies (PAA, + Lin and - Lin, Z3HA, E2HA) elicited significant upwind flight in Manduca females by itself (data not shown). Thus, we chose to test PAA and Z3HA against a synthetic 3-component mixture of three floral volatiles derived from Datura wrightii (benzyl alcohol 90\%, \pm linalool racemic mixture $7 \%$, benzaldehyde 3\%) shown to mediate flight and feeding behavior in Manduca males (Flower Mix, determined by Riffel et al., 2009). Note that the synthetic mixture already incor- 

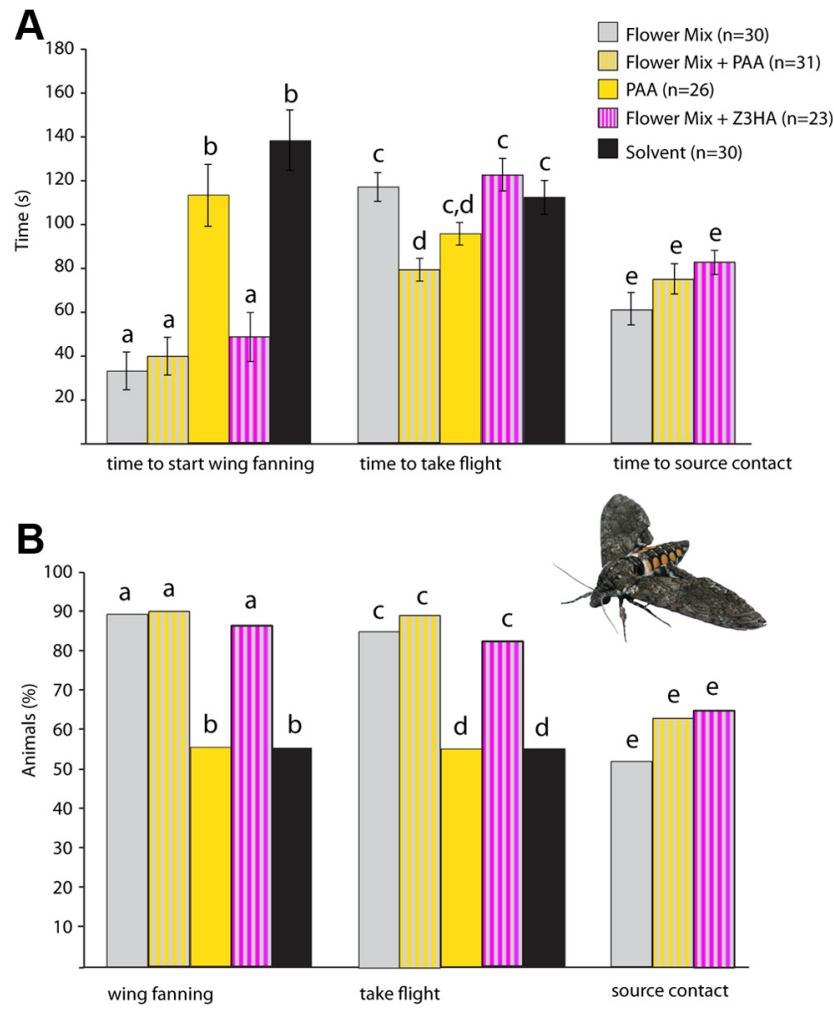

Figure 4. Behavioral correlates of AL network processing. The behavioral activity of PAA and Z3HA was assessed in a wind tunnel assay. We examined upwind flight and source contact behavior to a behaviorally attractive synthetic 3-component mixture (Flower Mix, determined by Riffell et al., 2009), with and without the addition of 1\% PAA or Z3HA to the mixture (Flower Mix, $n=30$, gray bars; Flower Mix + PAA, $n=31$, gray/yellow striped bars; PAA, $n=26$, yellow bars; Flower Mix + Z3HA, $n=23$, gray/magenta striped bars; Solvent (mineral oil) $n=$ 30 , black bars). $A$, Average time elapsed during olfactory behavior. Moths took flight significantly faster when PAA was added to the synthetic Flower Mix ( $p=0.01, t$ test), but not when PAA was presented alone. Substitution of another component (Z3HA; also compare Fig. 3) neither changed activity nor enhanced source contact in moths. $\boldsymbol{B}$, Percentages of moths displaying each behavioral characteristic tested. Nearly all (at least $85 \%$ total) animals that started wing fanning also took flight. Note that the number of source contacts ( $\sim 60 \%)$ was not affected by adding PAA or Z3HA. In contrast, PAA or the mineral oil control alone elicited wing fanning and flight in only $50 \%$ of animals tested with no source contact or oriented flight observed.

porated (-)-linalool, a compound providing high pattern similarity to our tested mixture (Fig. $3 A$ ).

140 animals were tested to five different stimuli (Fig. 4). At least $85 \%$ of female Manduca exhibited wing fanning and took flight to the Flower Mix either with or without PAA or Z3HA (Fig. $4 B$ ). However, moths took flight significantly faster when PAA was added to the synthetic flower mixture $(p=0.01, t$ test $)$, although the time needed to contact the source did not change. This indicates that PAA added to the behaviorally active mixture has no synergistic effect on either the probability of flight or net source contact, but significantly accelerates the initial take-off time for female moths. However, PAA and the mineral oil control alone elicited wing fanning and flight in only $\sim 50 \%$ of animals, with no source contact or oriented flight. In contrast, adding Z3HA to the Flower Mix (a compound exhibiting lower levels of signal modulation, Fig. 3), neither altered activity nor enhanced source contact in moths against the flower mix alone (Fig. 4). Thus, high levels of input-output modulation in the AL network for specific odors can correlate to behavioral output.

\section{Discussion}

Applying optical calcium imaging techniques, we directly compared the olfactory responses in individual female moths to an artificial host odor mixture and its single components simultaneously at two different processing levels (OSN input and PN output). We show that olfactory information in the AL undergoes intense network modulation that establishes a unique "mixture feature" separate from the simple sum of individual compound identities. The modulation of spatial activity patterns in the AL, most obvious for phenyl acetaldehyde, can also have potential implications for olfactory behavior in Manduca as assessed by flight tunnel analyses.

Input/output comparison reveals spatiotemporal modulation Recording odor-evoked activity to five monomolecular odors and their mixture showed that the resulting input mixture pattern could generally be predicted from the single component responses. This is in agreement with previous insect studies (Deisig et al., 2006; Carlsson et al., 2007; Silbering and Galizia, 2007). However, at the output level, strong nonlinear interactions that could not be predicted from the single component patterns occurred in six of seven animals, even when controlling for concentration (see Materials and Methods, Optophysiology, Odor stimulation). Four animals showed strong suppressive effects in the PN response (Fig. $2 B, D, E, G$ ), whereas synergism also occurred in two animals (Fig. $2 C$, recruitment in area 2 and 8; Fig. $3 F$, strong inhibition in region 7). Interestingly, in one case, both interactions were witnessed simultaneously (Fig. 2D, synergism/ recruitment in area 4 , suppression region 6 ). The observed variation between animals is most likely due to different focal planes used during recording. However, the strong nonlinear interactions observed at the output of six of seven animals support conclusions concerning the important role of the modulatory interneuron network in insects (Hildebrand et al., 1992; Chou et al., 2010; Lei et al., 2011; Reisenman et al., 2011; Kuebler et al., $2011 \mathrm{a}, \mathrm{b})$, and thus strengthen the idea of a novel feature percept generated by the AL. Moreover, these results are consistent with our previous electrophysiological study at the single neuron level (Kuebler et al., 2011b) and indicate that the mechanisms of mixture processing revealed at the single neuron level are maintained across the glomerular array in the AL.

In contrast to honeybees (Fonta et al., 1993), most local neurons in Manduca show a typical broad "symmetrical" arborization pattern innervating the majority of AL glomeruli (Matsumoto and Hildebrand, 1981; Kuebler et al., 2011b; Reisenman et al., 2011). Consequently, an inhibitory global network incorporating a population of neurons with glomerulus-specific connectivity for general odor mixtures seems unlikely in Manduca (but compare the Manduca MGC: Christensen and Hildebrand, 1997; Lei et al., 2002; Heinbockel et al., 2004). However, as stated before, our approach was not intended to reveal the mechanistic function of specific glomerulus-dependent interactions. Despite the strong interindividual differences within our dataset (Fig. 2, number of activity regions varies across animals, see text above), we found high levels of signal modulation from input to output between animals (Fig. 2). Our study thus reveals the capacity of the underlying neuronal network to generate mixturespecific representations. Observed suppressive effects (Fig. 2) may enhance the contrast of mixture representation (Sachse and Galizia, 2002; Vucinić et al., 2006) as well as enhance intraglomerular synchrony (Lei et al., 2002) and therefore build a profound basis for further downstream mixture processing in the insect's brain. Both spatial features and temporal kinetics of the 
network including neuronal spiking patterns and firing and oscillatory synchronicity (see review in Lei and Vickers, 2008) combine to develop the resultant output image of the mixture. However, the direct relationship between spatial and temporal mixture modulation was not evaluated here. Future studies, including pharmacological manipulation of the interneuron network through e.g., GABA-antagonists such as bicuculline (Lei et al., 2009), can help elucidate the underlying network mechanisms modulating AL output.

\section{Behavioral correlates and implications for spatiotemporal modulation}

Comparing patterns of odor representation at the two processing levels revealed increased network interactions for both the mixture and its specific components. Interestingly, phenyl acetaldehyde (PAA) elicited the most spatiotemporal modulation between input and output when compared with all other stimuli. Moreover, the network modulation of the PAA signal between processing levels is not only obvious during odor stimulation (Fig. 3A) but is reflected across the entire recording and particularly in the late post-odor response (Fig. 3B). The existence of a late post-odor response is challenging, as olfactory-guided behavior has been shown to be triggered within only a few ms after odor onset (Ditzen et al., 2003; Wright et al., 2009). In contrast to a recent study in honeybees (Szyszka et al., 2011), we show odorspecific post-odor response patterns at both the OSN and the PN processing levels (Fig. $3 B$ ) that could indicate a role for shortterm olfactory storage of working memory (Baddeley, 1992).

The decreased pattern correlation suggests that PAA establishes an unique representation in the AL from the other components tested. In addition, nearly all tested PNs and local interneurons (LNs) responded to PAA in our former electrophysiological study (Kuebler et al., 2011b). We thus questioned whether the unique AL processing of PAA has any implications for behavioral activity in Manduca sexta. PAA is a widespread plant odor, present in many green plant parts and flowers (Kessler and Baldwin, 2001), and could thus serve as a general host cue for Manduca sexta.

For both our previous electrophysiological study (Kuebler et al., 2011b) and the current optophysiological analyses, we chose a representative group of structurally diverse compounds known to be present in Manduca sexta host plants (Kessler and Baldwin, 2001; Allmann and Baldwin, 2010). As expected, our diverse mix of both floral and plant volatiles was not behaviorally active on its own. Hence, to assess the potential role for spatiotemporal signal modulation in the $\mathrm{AL}$, we tested two host compounds with varying levels of input-output modulation against a known behaviorally active host volatile mixture (Riffell et al., 2009a). In our behavioral study, the compound exhibiting the highest levels of signal modulation in optophysiological studies, PAA, did not enhance the probability of flight or source contact to the flower mixture, but significantly accelerated the time needed for female Manduca to take flight. Substitution of a component with lower levels of signal modulation (Z3HA, compare Fig. 3) neither changed activity, nor enhanced source contact in moths (Fig. 4).

Our behavioral and physiological results show a correlation between nonlinear mixture processing in the moth $\mathrm{AL}$ and olfactory-guided behavior. PAA not only exhibited high levels of spatiotemporal modulation against our tested mixture as well as between input and output, but also elicited responses across all single unit recordings of PNs and LNs in our previous study (Kuebler et al., 2011b). This broad network modulation, coupled with the observed increase in activity resulting from the addition of PAA to a behaviorally active mixture, suggests that PAA could serve as a general attention cue to "focus" the olfactory system to a potentially relevant odor source e.g., shown for cross-modal integration in humans (Gottfried and Dolan, 2003; Bensafi et al., 2007). In Gottfried and Dolan's study, participants detected odors faster and more accurately when they were presented with semantically congruent pictures compared with incongruent ones. Interestingly, this behavioral result was likewise reflected in enhanced neural activity, as in our current study. As all olfactory input must pass through the AL, any modulation of the output signal by the AL will have direct effects on subsequent olfactory processing. Although we do not yet fully understand how higher brain centers such as the mushroom bodies and lateral horn process this information, the diversity of AL modulation in our study suggests a network functionally adapted to meet the particular "olfactory world" of our species (Martin et al., 2011). In addition, while some behavior can be driven by single odors that do not require modulation, many behaviorally relevant odor cues are multicomponent and must be integrated in some form (Martin et al., 2011). Pharmacological manipulation of pheromone processing in the Manduca AL has also shown a direct impact on behavior (Lei et al., 2009). Our results indicate that the extent of integration can likewise correspond to varying levels of behavioral activity.

Our observed correlation between spatiotemporal patterning in the AL and behavior cannot conclusively link specific inputoutput modulation at the first synapse of the moth olfactory system through several synapses to resultant behavior. Further experiments, testing e.g., identical output activity patterns evoked by dissimilar odorants with different input channels may clarify the role of evoked neural activity patterns in establishing olfactory guided behavior.

\section{Conclusions}

Our results indicate that odor mixtures in the moth olfactory system establish a unique mixture percept separate from individual component identities as early as the first olfactory processing stage, the AL. Complex host mixture processing in the moth olfactory system is thus a highly nonlinear process that cannot be deduced from peripheral input. Moreover, our results indicate that the degree of network interactions in the AL is, to a certain extent, component-specific and can correlate to particular behavioral outputs. Indeed, our results show that female moths exhibited significantly accelerated behavioral activity when a compound with unique spatiotemporal response properties was added to an already behaviorally attractive odor mixture. To our knowledge, this is the first study providing evidence for an attention-like cue priming the olfactory network in insects. Future studies that continue to assess innate olfactory behavior along with quantitative assessment of spatiotemporal mixture patterns are essential to determine the role of these mixture and component "percepts" in the ultimate ethology of insects.

\section{References}

Allmann S, Baldwin IT (2010) Insects betray themselves in nature to predators by rapid isomerization of green leaf volatiles. Science 329:1075-1078.

Baddeley A (1992) Working memory. Science 255:556-559.

Bell RA, Joachim FG (1976) Techniques for rearing laboratory colonies of tobacco hornworms and pink bollworms. Ann Entomol Soc Am 69:365-373.

Bensafi M, Frasnelli J, Reden J, Hummel T (2007) The neural representation of odor is modulated by the presence of a trigeminal stimulus during odor encoding. Clinical Neurophysiology 118:696-701. 
Bhandawat V, Olsen SR, Gouwens NW, Schlief ML, Wilson RI (2007) Sensory processing in the Drosophila antennal lobe increases reliability and separability of ensemble odor representations. Nat Neurosci 10:1474-1482.

Carlsson MA, Chong KY, Daniels W, Hansson BS, Pearce TC (2007) Component information is preserved in glomerular responses to binary odor mixtures in the moth Spodoptera littoralis. Chem Senses 32:433-443.

Carlsson MA, Knüsel P, Verschure PF, Hansson BS (2005) Spatio-temporal $\mathrm{Ca}^{2+}$ dynamics of moth olfactory projection neurones. Eur J Neurosci 22:647-657.

Chou YH, Spletter ML, Yaksi E, Leong JC, Wilson RI, Luo L (2010) Diversity and wiring variability of olfactory local interneurons in the Drosophila antennal lobe. Nat Neurosci 13:439-449.

Christensen TA, Hildebrand JG (1997) Coincident stimulation with pheromone components improves temporal pattern resolution in central olfactory neurons. J Neurophysiol 77:775-781.

Christensen TA, Hildebrand JG (2002) Pheromonal and host-odor processing in the insect antennal lobe: how different? Curr Opin Neurobiol 12:393-399.

de Bruyne M, Baker TC (2008) Odor detection in insects: volatile codes. J Chem Ecol 34:882-897.

Deisig N, Lachnit H, Sandoz JC, Lober K, Giurfa M (2003) A modified version of the unique cue theory accounts for olfactory compound processing in honeybees. Learn Mem 10:199-208.

Deisig N, Giurfa M, Lachnit H, Sandoz JC (2006) Neural representation of olfactory mixtures in the honeybee antennal lobe. Eur J Neurosci 24:1161-1174.

Deisig N, Giurfa M, Sandoz JC (2010) Antennal lobe processing increases separability of odor mixture representations in the honeybee. J Neurophysiol 103:2185-2194.

Ditzen M, Evers JF, Galizia CG (2003) Odor similarity does not influence the time needed for odor processing. Chem Senses 28:781-789.

Duchamp-Viret P, Duchamp A, Chaput MA (2003) Single olfactory sensory neurons simultaneously integrate the components of an odour mixture. Eur J Neurosci 18:2690-2696.

Fonta C, Sun X-J, Masson C (1993) Morphology and spatial distribution of bee antennal lobe interneurones responsive to odours. Chem Senses 18:101-119.

Fraser AM, Mechaber WL, Hildebrand JG (2003) Electroantennographic and behavioral responses of the sphinx moth Manduca sexta to host plant headspace volatiles. J Chem Ecol 29:1813-1833.

Fried HU, Fuss SH, Korsching SI (2002) Selective imaging of presynaptic activity in the mouse olfactory bulb shows concentration and structure dependence of odor responses in identified glomeruli. Proc Natl Acad Sci U S A 99:3222-3227.

Friedrich RW, Korsching SI (1998) Chemotopic, combinatorial, and noncombinatorial odorant representations in the olfactory bulb revealed using a voltage-sensitive axon tracer. J Neurosci 18:9977-9988.

Galili DS, Lüdke A, Galizia CG, Szyszka P, Tanimoto H (2011) Olfactory trace conditioning in Drosophila. J Neurosci 31:7240-7248.

Galizia CG, Rössler W (2010) Parallel olfactory systems in insects: anatomy and function. Annu Rev Entomol 55:399-420.

Galizia CG, Joerges J, Faber T, Küttner A, Hölldobler B, Menzel R (1998) Functional optical imaging of olfactory glomeruli in bees and ants. A universal code? Eur J Neurosci 10 [Suppl 10]:435.

Galizia CG, Menzel R, Holldobler B (1999a) Optical imaging of odorevoked glomerular activity patterns in the antennal lobes of the ant Camponotus rufipes. Naturwissenschaften 86:533-537.

Galizia CG, Sachse S, Rappert A, Menzel R (1999b) The glomerular code for odor representation is species specific in the honeybee Apis mellifera. Nat Neurosci 2:473-478.

Gottfried JA, Dolan RJ (2003) The nose smells what the eye sees: crossmodal visual facilitation of human olfactory perception. Neuron 39:375-386.

Grosse-Wilde E, Kuebler LS, Bucks S, Vogel H, Wicher D, Hansson BS (2011) Antennal transcriptome of Manduca sexta. Proc Natl Acad Sci U S A 108:7449-7454.

Hansson BS, Anton S (2000) Function and morphology of the antennal lobe: new developments. Annu Rev Entomol 45:203-231.

Hansson BS, Carlsson MA, Kalinovà B (2003) Olfactory activation patterns in the antennal lobe of the sphinx moth, Manduca sexta. J Comp Physiol A Neuroethol Sens Neural Behav Physiol 189:301-308.

Heinbockel T, Christensen TA, Hildebrand JG (2004) Representation of bi- nary pheromone blends by glomerulus-specific olfactory projection neurons. J Comp Physiol A Neuroethol Sens Neural Behav Physiol 190:1023-1037.

Hildebrand JG, Shepherd GM (1997) Mechanisms of olfactory discrimination: converging evidence for common principles across phyla. Annu Rev Neurosci 20:595-631.

Hildebrand JG, Christensen TA, Harrow ID, Homberg U, Matsumoto SG, Waldrop BR (1992) The roles of local interneurons in the processing of olfactory information in the antennal lobes of the moth Manduca sexta. Acta Biol Hung 43:167-174.

Hillier NK, Vickers NJ (2011) Mixture interactions in moth olfactory physiology: examining the effects of odorant mixture, concentration, distal stimulation, and antennal nerve transection on sensillar responses. Chem Senses 36:93-108.

Homberg U, Montague RA, Hildebrand JG (1988) Anatomy of antennocerebral pathways in the brain of the sphinx moth Manduca sexta. Cell Tissue Res 254:255-281.

Homberg U, Christensen TA, Hildebrand JG (1989) Structure and function of the deutocerebrum in insects. Annu Rev Entomol 34:477-501

Joerges C, Neyer J (1997) Transforming strategic interaction into deliberative problem-solving: European comitology in the foodstuffs sector. J Eur Public Policy 4:609-625.

Joerges J, Küttner A, Galizia CG, Menzel R (1997) Representations of odours and odour mixtures visualized in the honeybee brain. Nature 387:285-288.

Kessler A, Baldwin IT (2001) Defensive function of herbivore-induced plant volatile emissions in nature. Science 291:2141-2144.

King JR, Christensen TA, Hildebrand JG (2000) Response characteristics of an identified, sexually dimorphic olfactory glomerulus. J Neurosci 20:2391-2399.

Kuebler LS, Olsson SB, Hansson BS (2011a) First order processing of complex olfactory information in the moth brain. Procedia Comput Sci 7:258-260

Kuebler LS, Olsson SB, Weniger R, Hansson BS (2011b) Neuronal processing of complex mixtures establishes a unique odor representation in the moth antennal lobe. Front Neural Circuits 5:7.

Lei H, Vickers N (2008) Central processing of natural odor mixtures in insects. J Chem Ecol 34:915-927.

Lei H, Christensen TA, Hildebrand JG (2002) Local inhibition modulates odor-evoked synchronization of glomerulus-specific output neurons. Nat Neurosci 5:557-565.

Lei H, Riffell JA, Gage SL, Hildebrand JG (2009) Contrast enhancement of stimulus intermittency in a primary olfactory network and its behavioral significance. J Biol 8:21.

Lei H, Reisenman CE, Wilson CH, Gabbur P, Hildebrand JG (2011) Spiking patterns and their functional implications in the antennal lobe of the tobacco hornworm Manduca sexta. PLoS One 6:e23382.

Mantel N (1967) The detection of disease clustering and a generalized regression approach. Cancer Res 27:209-220.

Martin JP, Beyerlein A, Dacks AM, Reisenman CE, Riffell JA, Lei H, Hildebrand JG (2011) The neurobiology of insect olfaction: sensory processing in a comparative context. Prog Neurobiol 95:427-447.

Matsumoto S, Hildebrand J (1981) Olfactory mechanisms in the moth Manduca sexta: response characteristics and morphology of central neurons in the antennal lobes. Proc R Soc Lond B Biol Sci 213:249-277.

Meister M, Bonhoeffer T (2001) Tuning and topography in an odor map on the rat olfactory bulb. J Neurosci 21:1351-1360.

Olsson SB, Kuebler LS, Veit D, Steck K, Schmidt A, Knaden M, Hansson BS (2011) A novel multicomponent stimulus device for use in olfactory experiments. J Neurosci Methods 195:1-9.

R Development Core Team (2011) R: A language and environment for statistical computing. R Foundation for Statistical Computing, Vienna. http://www.R-project.org/.

Reinhard J, Sinclair M, Srinivasan MV, Claudianos C (2010) Honeybees learn odour mixtures via a selection of key odorants. PLoS One 5:e9110.

Reisenman CE, Christensen TA, Francke W, Hildebrand JG (2004) Enantioselectivity of projection neurons innervating identified olfactory glomeruli. J Neurosci 24:2602-2611.

Reisenman CE, Dacks AM, Hildebrand JG (2011) Local interneuron diversity in the primary olfactory center of the moth Manduca sexta. J Comp Physiol A Neuroethol Sens Neural Behav Physiol 197:653-665.

Riffell JA, Lei H, Hildebrand JG (2009a) Inaugural Article: Neural correlates 
of behavior in the moth Manduca sexta in response to complex odors. Proc Natl Acad Sci U S A 106:19219-19226.

Riffell JA, Lei H, Christensen TA, Hildebrand JG (2009b) Characterization and coding of behaviorally significant odor mixtures. Curr Biol 19:335-340.

Rubin BD, Katz LC (1999) Optical imaging of odorant representations in the mammalian olfactory bulb. Neuron 23:499-511.

Sachse S, Galizia CG (2002) Role of inhibition for temporal and spatial odor representation in olfactory output neurons: a calcium imaging study. J Neurophysiol 87:1106-1117.

Sachse S, Galizia CG (2003) The coding of odour-intensity in the honeybee antennal lobe: local computation optimizes odour representation. Eur J Neurosci 18:2119-2132.

Sandoz JC, Galizia CG, Menzel R (2003) Side-specific olfactory conditioning leads to more specific odor representation between sides but not within sides in the honeybee antennal lobes. Neuroscience 120: 1137-1148.

Schlief ML, Wilson RI (2007) Olfactory processing and behavior downstream from highly selective receptor neurons. Nat Neurosci 10: $623-630$.

Shields VD, Hildebrand JG (2000) Responses of a population of antennal olfactory receptor cells in the female moth Manduca sexta to plantassociated volatile organic compounds. J Comp Physiol A Neuroethol Sens Neural Behav Physiol 186:1135-1151.

Silbering AF, Galizia CG (2007) Processing of odor mixtures in the Drosoph- ila antennal lobe reveals both global inhibition and glomerulus-specific interactions. J Neurosci 27:11966-11977.

Skiri HT, Galizia CG, Mustaparta H (2004) Representation of primary plant odorants in the antennal lobe of the moth Heliothis virescens using calcium imaging. Chem Senses 29:253-267.

Smith BH (1998) Analysis of interaction in binary odorant mixtures. Physiol Behav 65:397-407.

Su CY, Martelli C, Emonet T, Carlson JR (2011) Temporal coding of odor mixtures in an olfactory receptor neuron. Proc Natl Acad Sci U S A 108:5075-5080.

Szyszka P, Demmler C, Oemisch M, Sommer L, Biergans S, Birnbach B, Silbering AF, Galizia CG (2011) Mind the gap: olfactory trace conditioning in honeybees. J Neurosci 31:7229-7239.

Uchida N, Takahashi YK, Tanifuji M, Mori K (2000) Odor maps in the mammalian olfactory bulb: domain organization and odorant structural features. Nat Neurosci 3:1035-1043.

Vucinić D, Cohen LB, Kosmidis EK (2006) Interglomerular centersurround inhibition shapes odorant-evoked input to the mouse olfactory bulb in vivo. J Neurophysiol 95:1881-1887.

Wang JW, Wong AM, Flores J, Vosshall LB, Axel R (2003) Two-photon calcium imaging reveals an odor-evoked map of activity in the fly brain. Cell 112:271-282.

Wright GA, Carlton M, Smith BH (2009) A honeybee's ability to learn, recognize, and discriminate odors depends upon odor sampling time and concentration. Behav Neurosci 123:36-43. 